\section{Summary}

\title{
Phenomics data processing: Extracting temperature dose-response curves from repeated measurements
}

\author{
Lukas Roth ${ }^{\mathrm{a}, *}$, Hans-Peter Piepho ${ }^{\mathrm{b}}$, Andreas Hund ${ }^{\mathrm{a}}$ \\ ${ }^{a}$ ETH Zurich, Institute of Agricultural Sciences, Universitätstrasse 2, 8092 Zurich, Switzerland \\ ${ }^{b}$ University of Hohenheim, Institute for Crop Science, Biostatistics Unit, Fruwirthstrasse 23, 70593 Stuttgart, Germany
}

\begin{abstract}
Temperature is a main driver of plant growth and development. New phenotyping tools enable quantify-
\end{abstract} ing the temperature response of hundreds of genotypes. Yet, particularly for field-derived data, the process of temperature response modelling bears potential flaws and pitfalls with regard to the interpretation of derived parameters. In this study, climate data from three growing seasons with differing temperature distributions served as starting point for a wheat stem elongation growth simulation, based on a four-parametric Wang-Engel temperature response function. To extract dose-responses from the simulated data, a novel approach to use temperature courses with high temporal resolution was developed. Linear and asymptotic parametric modelling approaches to predict the cardinal temperatures were investigated. An asymptotic model extracted the base and optimum temperature of growth and the maximum growth rate with high precision, whereas simpler, linear models failed to do so. However, when including seasonally changing cardinal temperatures, the prediction accuracy of the asymptotic model was strongly reduced. We conclude that using an asymptotic model based on temperature courses with high temporal resolution is suitable to extract meaningful parameters from field-based data. Consequently, applying the presented modelling approach to high-throughput phenotyping data of breeding nurseries may help selecting for climate suitability.

Keywords: growth, high-throughput field phenotyping, stem elongation, wheat (Triticum aestivum).

\section{Introduction}

Already in 1996, van Haren noted that the knowledge of crop responses to weather extremes is incomplete and not explicitly included in crop models. Today, high-throughout field phenotyping (HTFP) potentially enables to quantify crop growth—and consequently crop response-of hundreds of genotypes under field conditions (Araus et al., 2018). Understanding crop responses to environmental factors is essential to secure global food demands: Improvement in wheat yields stagnated on a global scale in the past three decades (Brisson et al., 2010; Laidig

*Corresponding author: lukas.roth@usys.ethz.ch 
et al., 2017). It was suspected that for Europe, the changing climate increasingly impacts wheat yields (Brisson et al., 2010). Mitigating these changes in plant breeding to improve yield requires understanding crop responses to environmental factors (Ramirez-Villegas et al., 2015). A main driver of plant growth and development is temperature (Porter and Gawith, 1999).

\subsection{Approaches to quantify temperature responses under controlled conditions}

The effects of changes in temperature on crops are well studied in controlled environments, but the translation of insights to the field is not straightforward. The temperature response of developmental processes is usually studied by exposing plants to different temperature regimes-often during rather short time period of their life cycle. Two different approaches are used: one can either (1) expose different plants of the same genotype to different temperatures (Slafer and Rawson, 1995; Hund et al., 2012; Reimer et al., 2013), or (2) apply short phases of different temperatures to the same plant. Examples of the latter approach are the studies on leaf growth performed in the indoor platform 'Phenoarch', summarized by Parent and Tardieu (2012). This approach requires that the temperature response is measured at a constant growth phase, during which growth is linear when the temperature remains constant. Hence, response-pattern can be observed non-destructively on the same plant, offering a high throughput. In such experiments, the conditions of all but one covariate may be kept constant. Furthermore, the reversibility of the process can be tested by repeatedly applying the same conditions during the course of the experiment.

In general, developmental processes follow some sort of peak function in response to increasing temperatures, i.e., there is a base temperature $T_{\min }$ at which growth starts, an optimal temperature $T_{\mathrm{opt}}$ at which growth reaches its maximum rate $r_{\max }$, and a maximum temperature $T_{\mathrm{opt}}$ at which growth stops (Porter and Gawith, 1999). Depending on the research field, different models are used to approximate such functions-for an overview see, e.g., Wang et al. (2017); Parent et al. (2019) for plants, and Rebaudo et al. (2018); Rebaudo and Rabhi (2018) for ectoterms (i.e., animals whose regulation of body temperature depends on external sources). In their work, Wang et al. (2017) distinguish four types of temperature response functions with increasing complexity. Clearly, type 4 functions (linear or curvilinear functions with three cardinal temperatures) are most appropriate to describe temperature responses. Among the most widely used type 4 functions for plants are the Wang-Engel cardinal temperature function (Wang et al., 2017) and a modified version of the reaction rates equation from Johnson et al. (1942) applied to different growth processes in a variety of major crops by Parent and Tardieu (2012). We will refer to these different response functions in detail further down.

\subsection{Cardinal temperatures of wheat}

For wheat, meta-analyses summarizing cardinal temperatures of different developmental processes are to some extent controversial. Porter and Gawith (1999) summarized 65 studies with regard to the minimum $\left(T_{\min }\right)$, optimum $\left(T_{\text {opt }}\right)$ and maximum $\left(T_{\max }\right)$ temperature. Mean cardinal temperatures for shoot growth were $3.0{ }^{\circ} \mathrm{C}$ $\left(T_{\min }\right), 20.3{ }^{\circ} \mathrm{C}\left(T_{\mathrm{opt}}\right)$ and $>20.9{ }^{\circ} \mathrm{C}\left(T_{\max }\right)$. Notably, $T_{\mathrm{opt}}$ and $T_{\max }$ were very close together in many studies. 
In contrast to the comparably low $T_{\text {opt }}$ summarized by Porter and Gawith (1999), Parent and Tardieu (2012) reported a significantly higher $T_{\text {opt }}$ of $27.7^{\circ} \mathrm{C}$ when summarizing 8 studies and own data. We speculate that the discrepancies between the two studies are caused by the fact that the former is based mainly on studies using long-term exposure of individual plants, and the latter on studies that applied short-term changes in temperatures on a growing organ. In the present study we will use the more conservative, i.e., lower, temperature optimum reported by Porter and Gawith (1999).

There is evidence that cardinal temperatures are not constant during the lifetime of a crop: Slafer and Rawson (1995) examined $T_{\min }$ and $T_{\text {opt }}$ for leaf appearance rates of vernalized winter wheat plants within three phases, i) after vernalization up to the terminal spikelet, ii) from the terminal spikelet up to heading, and iii) from heading to anthesis. The authors found that, averaged across cultivars, $T_{\min }$ was $-1.9^{\circ} \mathrm{C}, 1.2{ }^{\circ} \mathrm{C}$ and $8.1{ }^{\circ} \mathrm{C}$, while $T_{\text {opt }}$ was $<22{ }^{\circ} \mathrm{C}, 25{ }^{\circ} \mathrm{C}$ and $>25{ }^{\circ} \mathrm{C}$ for the three different phases, respectively. In addition, the cultivars differed substantially for the two parameters. Consequently, one may assume that particularly the base temperature may change cultivar-specific throughout the season. Nevertheless, the estimates from Slafer and Rawson (1995) of cardinal temperatures and their changes were derived from a controlled environment study. The benefits of controlled environment phenotyping lies in the ability to test a wide range of temperature regimes while keeping other influential factors, such as vapor pressure deficit and light, constant. However, plants usually do not experience such conditions in the field, as discussed by Slafer and Rawson (1995).

\subsection{Approaches to quantify temperature responses with high-throughput in the field}

Under field conditions, the temperature regimes are distinctly different from the controlled-environment studies considered so far. The environmental covariates in the field follow an annual and diurnal pattern to which plants adjust their life cycle. HTFP offers the possibility to assess crop growth under such more realistic conditions with high temporal and spatial resolution (Rebetzke et al., 2019). Hence, one can use the temperature course during the season to quantify the genotype-specific temperature responses. Still, such studies are sparse. Grieder et al. (2015) extracted the base temperature and response to increasing temperatures at early growth stages before terminal spikelet formation. The authors were able to extract heritable slopes and intercepts of linear responses. Kronenberg et al. (2020a) were able to extract heritable response traits for the stem elongation phase based on data from three seasons. Again, the authors used linear regressions of growth versus temperature as model. Kronenberg et al. (2020a) argued that the recorded average temperatures in the measuring intervals were well below the optimum temperature of $27.7^{\circ} \mathrm{C}$ reported by Parent and Tardieu (2012), which would justify approximating a curvilinear with a linear function. However, the optimum temperature of $20.3{ }^{\circ} \mathrm{C}$ summarized by Porter and Gawith (1999) is distinctly lower. Following those results, it is likely that growth reached its optimum during several hours of the day during their measurement period. Therefore, the extracted temperature response (slope) may be greatly affected by the optimum temperature and maximum growth rate at this temperature. Thus, modelling plant response to temperature based on HTFP-derived data bears several challenges, e.g., 
the comparably sparse measuring frequency, the unpredictable range of temperatures, the changes of cardinal temperatures during the measuring period, and confounding effects between earliness and seasonal temperature development.

\subsection{Research aims}

HTFP data are characterized by irregular and long trait measurement intervals but regular and short covariate measurement intervals. Our main research question was how to parameterize genotype-specific temperature response pattern from such HTFP data. Using the stem elongation phase of winter wheat as an example, we aimed to evaluate a new modelling approach with regard to its suitability. Therefore, we simulated the temperatureresponse in the stem elongation phase using a Wang-Engel response function. The chosen parameters were based on existing literature on cardinal temperatures in winter wheat (Porter and Gawith, 1999; Parent and Tardieu, 2012) and enhanced with realistic temperature recordings of the stem elongation phase at the FIP site (Kronenberg et al., 2020a). To evaluate the strengths and limitations of our approach, we added changing cardinal temperatures, shifts in the start and end of the elongation phase, measurement noise, and spatial field inhomogeneities to our simulation.

\section{Background}

\subsection{Parametric versus semi-parametric approaches}

Semi-parametric approaches (e.g., using P-splines) are often favored to extract traits related to growth processes from HTFP time series (van Eeuwijk et al., 2019), as they avoid the daunting task of modeling explicitly the-potentially unknown-influence of time-dependent covariate courses. In a previous research, we have shown that semi-parametric approaches are suitable to extract timing of key stages and quantities at defined time points or periods from simulated HTFP data. Those data were generated using a dose-response curve that was unknown to the model being fitted, and corresponding covariate dependencies masked by a "smoothing" part in the model (Roth et al., 2021).

In contrast, to extract temperature responses, one cannot avoid modeling a dose-response curve explicitly, as one is interested in its parameters. Consequently, we hereafter propose a parametric approach. Although our focus is on HTFP data, the approach is also directly applicable to indoor platform data, but additionally considers HTFP specific characteristics.

In comparison to greenhouse or climate chamber data, HTFP data have two major drawbacks: (i) Field-based measurements are notoriously noisy due to environmental and soil inhomogeneities and measurement errors; (ii) the suitability of measured trait time series and covariate courses to answer a research question depends on the good luck of the scientist to catch a meaningful season. Research in agriculture has already addressed some of these limitations with the development of highly specialized experimental designs and matching statistical analysis. We could already show that following these principles is of advantage for HTFP as well (Roth et al., 
2021). For brevity, we therefore focus on the extraction of response traits from time series but refrain from further processing to adjusted genotype means. To allow determining and comparing the performance of non-linear and linear models, we use a simulation that is parametrized based on real field data.

\subsection{Temperature response functions}

The linear temperature response model used in Grieder et al. (2015) and Kronenberg et al. (2020a) is defined as

$$
r_{\text {linear }}(T)=a \cdot\left(T-T_{\min }\right),
$$

where $T$ is the temperature, $a$ is the slope of the response, and $T_{\min }$ the base temperature. Note that such a model causes negative growth rates $r$ for $T<T_{\min }$ and does not include an optimum temperature $T_{\text {opt }}$. Therefore, it is likely that the extracted temperature response (slope) is affected by the optimum temperature and maximum growth rate at this temperature. Additionally, using such a Type 1 response will come to its limitation when data ranges span a whole growing season where temperatures also expand to supra-optimal ranges (Parent et al., 2019). In the crop model community, models describing the response to temperature vary widely, but most of them consider an optimum temperature beyond which the growth rate levels off (Bonhomme, 2000; Parent et al., 2010; Wang et al., 2017).

Arrhenius type functions were shown to adequately describe the dose-response relationship between temperature and growth across a wide range of temperatures and species (Parent et al., 2010; Parent and Tardieu, 2012). However, it was disputed whether this approach allows for a mechanistic interpretation of the different model parameters (Clavijo Michelangeli et al., 2016). Evidently, research in wheat mainly focused on determining cardinal temperatures such as the lower and upper base temperature of growth $T_{\min }$ and $T_{\max }$ and the optimum temperature $T_{\text {opt }}$ where the growth rate $r$ is maximal (Porter and Gawith, 1999). Wang et al. (2017) could show that using the Wang-Engel temperature function (Wang and Engel, 1998) allows to adequately replace an Arrhenius type functions to describe growth rates using these cardinal temperatures,

$$
\begin{aligned}
& r_{\text {Wang-Engel }}(T)=r_{\max } \cdot\left(\frac{\left(2\left(T-T_{\min }\right)^{\alpha}\left(T_{\mathrm{opt}}-T_{\min }\right)^{\alpha}-\left(T-T_{\min }\right)^{2 \alpha}\right.}{\left(T_{\mathrm{opt}}-T_{\min }\right)^{2 \alpha}}\right)^{\beta} \\
& \alpha=\frac{\ln (2)}{\ln \left(\left(T_{\max }-T_{\min }\right) /\left(T_{\mathrm{opt}}-T_{\min }\right)\right)} \\
& \beta=1,
\end{aligned}
$$

where $T$ is the temperature, $r_{\max }$ the maximum growth rate at the temperature optimum $T_{\mathrm{opt}}, T_{\min }$ the lower base temperature and $T_{\max }$ the upper base temperature of growth.

The Wang-Engel temperature function is a Type 4 response (Wang et al., 2017) in regards that it is based on three cardinal temperatures $T_{\min }, T_{\max }$ and $T_{\mathrm{opt}}$. If aiming to extract genotype-specific cardinal temperatures as well as the maximum growth rate $r_{\max }$ from HTFP data, this would require to fit a four-parametric non-linear 
model with parameters that have interdependent constrains (e.g., $T_{\min }<T_{\text {opt }}$ ). In our experience, extracting meaningful parameters by fitting such a model to noisy HTFP data is practically impossible for different reasons, i.e., (i) the optimum temperature $T_{\text {opt }}$ and maximum growth rate $r_{\max }$ are closely correlated, (ii) for field experiments performed in target environments (without stress treatments), the maximum temperature $T_{\max }$ is typically outside the range of observed data, and (iii) proximity of cardinal temperatures $T_{\min }$ and $T_{\text {opt }}$ may prevent model conversions. Some authors have reacted to these restrictions by fixing one or multiple parameters and normalizing growth rates (Parent and Tardieu, 2012).

As a previously unconsidered alternative, we propose a Type 2 response that integrates an optimum temperature (Wang et al., 2017) to approximate the Arrhenius type functions of Parent and Tardieu (2012) based on an asymptotic model (Figure 1c),

$$
\begin{aligned}
& r=r_{\max } \cdot\left(1-\exp \left(-\exp (l r c) \cdot\left(T-T_{\min }\right)\right)\right) \\
& r_{\text {asym }}(T)= \begin{cases}r, & r>0 \\
0, & \text { otherwise }\end{cases}
\end{aligned}
$$

where $T$ is the temperature, $r_{\max }$ is the maximum growth rate (and therefore the asymptote of the curve), $T_{\min }$ the base temperature where the growth rate is zero, and $\operatorname{lrc}$ characterizes the steepness of the response as the natural logarithm $(l)$ of the rate $(r)$ constant $(c)$, thus $l r c$. The proposed asymptotic model does not consider a maximum temperature and therefore supra-optimal range, and has no inflection point (Paine et al., 2012). It requires to fit a three-parametric non-linear model.

2.3. Combining irregular and long trait measurement intervals with constant and short covariate measurement intervals

Independent of the specific dose-response model, one can describe a phenotype $y$ at time point $t$ (Figure 1a) as the result of such a dose-response model $r\left(\right.$ ) (Figure 1c) dependent on a temperature course $T_{t}$ (Figure 1b)

(a) Trait measurement

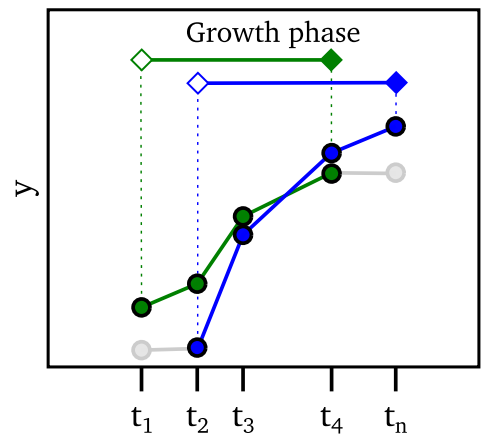

(b) Covariate measurement

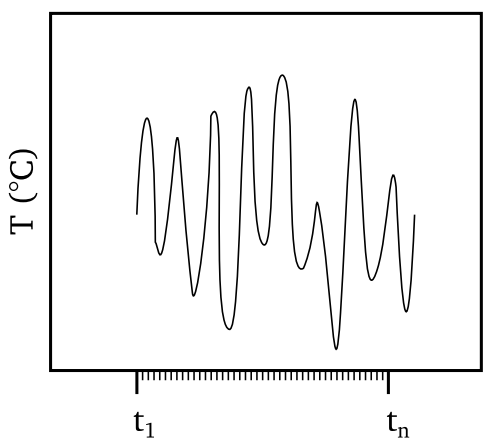

(c) Dose-response curve

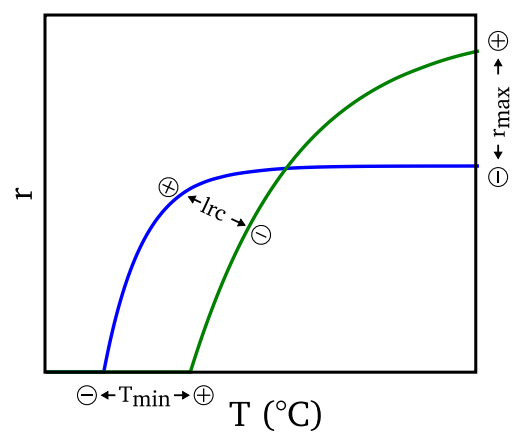

Figure 1: Schematic drawing of trait measurements with irregular and long intervals (a), covariate measurements with short and constant intervals (b), and the asymptotic dose-response model based on a maximum growth rate $\left(r_{\max }\right)$, minimum temperature $\left(T_{\min }\right)$, and steepness of the response $(\operatorname{lrc})(\mathrm{c})$. 
and a genotype-specific set of crop model parameters $\theta$, e.g., $\theta=\left(T_{\min }, T_{\mathrm{opt}}, T_{\max }, r_{\max }\right)$,

$$
y_{t}=\int_{t_{0}}^{t} r\left(T_{t^{\prime}}, \theta\right) d t^{\prime},
$$

where $t_{0}$ is the time point were growth started and therefore a timing of key stage trait that needs to be determined beforehand. Trait and covariates such as canopy height and temperatures are only measured at certain points in time, dependent on the measurement interval $\Delta m$. We therefore may discretize Equation 7 to

$$
y_{t}=\sum_{t^{\prime}=t_{0}}^{t} r\left(T_{t^{\prime}}, \theta\right) \cdot \Delta m,
$$

where $t^{\prime}$ are measurement time points with $\left(t^{\prime}=t_{0}, t_{0}+\Delta m, t_{0}+2 \Delta m, \ldots, t\right)$, e.g., days.

Note that fitting Equation 8 to data implies the same measurement interval for traits $y_{t}$ and temperatures $T_{t^{\prime}}$. Although the goal of HTFP is to measure at small measurement intervals, unfavorable weather conditions may prevent regular measurements at all times, while registering covariates is weather-independent and done with constant high frequency. Outdoor phenotyping therefore produces trait measurements with irregular and long measurement intervals $\Delta m_{d}$ (e.g., measurements every 3-4 days) but covariate measurements with constant and short measurement intervals $\Delta m_{h}$ (e.g., hourly measurements). Therefore, before applying Equation 8 to data, an aggregation step is required.

A common technique is to align trait and covariate measurements by aggregating the covariate values to a mean (e.g., Kronenberg et al., 2017, 2020a),

$$
y_{t}=\sum_{d=t_{0}}^{t}\left(r\left(\bar{T}_{d}, \theta\right) \cdot \Delta m_{d}\right),
$$

where $\bar{T}_{d}$ is the mean of all covariate measurements made in the time period between successive trait measurement time points $d$ where $\left(d=t_{0}, t_{0}+\Delta m_{1}, t_{0}+\Delta m_{1}+\Delta m_{2}, \ldots, t\right)$. In this study, we propose an alternative approach: One may also apply the dose-response function $r$ to each covariate value $T_{d h}$ where $h$ indexes covariate values between successive trait measurement time points $d,\left(h=1, \ldots, n_{d}\right)$, and sum up the responses to the trait measurements,

$$
y_{t}=\sum_{d=t_{0}}^{t}\left(\sum_{h=1}^{n_{d}} r\left(T_{d h}, \theta\right) \cdot \Delta m_{h}\right) .
$$

In the following, the first method is denoted the $T_{\text {mean }}$ method and the second method the $T_{\text {course }}$ method.

\section{Materials and Methods}

\subsection{Simulation of canopy height data}

The canopy growth of wheat genotypes was simulated using a growth function $g_{T}$ that was based on the Wang-Engel dose-response model of Equation 4 parameterized with four genotype-specific dose-response crop 
model parameters $\theta^{C}=\left(T_{\min }, T_{\mathrm{opt}}, T_{\max }, r_{\max }\right)$. This model was extended with time points for the start and end of growth, parameterized with two genotype-specific timing traits $\theta^{T}=\left(t \mathrm{PH}_{\text {start }}, t \mathrm{PH}_{\text {stop }}\right)$. An environment inhomogeneity factor $u$ and a measurement error $e$ were added, leading to the model

$$
y_{i t}=g_{T}\left(t, \theta_{i}^{C}, \theta_{i}^{T} ; T_{d h}\right)+u_{i t}+e_{t},
$$

where $y_{i t}$ is the measured canopy height (phenotype) for the $i$ th genotype at campaign time points $t$ in intervals of three days $(t=1,4,7, \ldots, T), \theta_{i}^{C}$ and $\theta_{i}^{T}$ are genotype-specific crop growth model parameters respectively timing traits, $T_{d h}$ are hourly $(h)$ temperature readings nested in measurement days $(d), u_{i t}$ a time point and genotype-specific inhomogeneity error (i.e., simulating the influence of other covariates than temperature) and $e_{t}$ a time point specific measurement error (i.e., simulating random measurement errors). The growth function $g_{T}$ was specified as an implementation of Equation 10 based on the Wang-Engel function of Equation 4,

$$
g_{T}\left(t, \theta^{C}, \theta^{T} ; T_{d h}\right)=\sum_{d=1}^{t} \begin{cases}\sum_{h=1}^{72} r_{\text {Wang-Engel }}\left(T_{d h}, \theta^{C}\right) & t \mathrm{PH}_{\text {start }}<d<t \mathrm{PH}_{\text {stop }} \\ 0, & \text { otherwise } .\end{cases}
$$

The genotype-specific traits $\theta_{i}^{C}$ and $\theta_{i}^{T}$ and the errors $u_{i t}$ and $e_{i t}$ were simulated using normal distributions $\left(\sim \mathcal{N}\left(\mu, \sigma^{2}\right)\right.$ ) for genotype traits respectively first order auto-correlations $\left(\sim \operatorname{AR}_{1}(\sigma, \rho)\right)$ for the errors. Specific $\mu$ and $\sigma^{2}$ were chosen based on literature if available, and otherwise based on own field data (Roth, 2021). Corresponding sources and chosen distributions for all simulation input parameter are summarized in Table 1. Two alternative simulations were performed: One with changing cardinal temperatures with time (indicated with ' $\rightarrow$ ' in Table 1), and one with fixed cardinal temperatures (set to the mean of changing cardinal temperatures indicated in Table 1).

Canopy growth was simulated for a measurement interval of three days for a period between the 15th of March and 20th of July based on temperature courses of three years (Figure 2). A total of 1,000 differing genotypes were assumed, resulting in 3,000 genotype time series. As temperature data source, real weather data of three consecutive years at the ETH research station of agricultural sciences in Lindau Eschikon, Switzerland (47.449 N, 8.682 E, 556 m a.s.l.) were used.

\subsection{Extracting dose-response curves}

To extract the Wang-Engel dose-response curve parameters $T_{\min }, T_{\text {opt }}$ and $r_{\max }$, the linear and asymptotic growth response models (Equation 1 and 6) were fitted to canopy height data between $t \mathrm{PH}_{\text {start }}$ and $t \mathrm{PH}_{\text {stop }}$ for two covariate options, $T_{\text {mean }}$ based on averaged temperatures between measurements (Equation 9), and $T_{\text {course }}$ based on temperature courses with a period of one hour (Equation 10).

Maximum-likelihood optimization was used to fit Equation 9 to a set of $t$ data points, $X_{t}=\left(\bar{T}_{t}, y_{t}-y_{(t-1)}\right)$. Fitting Equation 10 to data required modifying the definition of the data set so that $X_{t}=\left(\vec{T}_{t}, y_{t}-y_{(t-1)}\right)$, where $\vec{T}_{t}$ is a vector of covariate readings between trait measurement time points $t-1$ and $t$. 
Table 1: Model input parameters for the simulation.

\begin{tabular}{|c|c|c|c|}
\hline & & Values & Source \\
\hline$\theta_{i}^{T}$ & $\mathscr{N}\left(\mu, \sigma^{2}\right)$ & $\begin{array}{l}\mu_{T_{\min }}=1.5 \rightarrow 9.5^{\circ} \mathrm{C} \\
\sigma_{T_{\min }}=2{ }^{\circ} \mathrm{C} \\
\mu_{T_{\text {opt }}}=10.6 \rightarrow 21^{\circ} \mathrm{C} \\
\sigma_{T_{\text {opt }}}=2{ }^{\circ} \mathrm{C} \\
\mu_{T_{\max }}=30 \rightarrow 35^{\circ} \mathrm{C}{ }^{\circ} \mathrm{C} \\
\sigma_{T_{\max }}=2{ }^{\circ} \mathrm{C} \\
\mu_{r_{\max }}=1.05 \mathrm{~mm} \mathrm{~h}^{-1}, \sigma_{r_{\max }}=0.12 \mathrm{~mm} \mathrm{~h}^{-1}\end{array}$ & $\begin{array}{l}\text { Porter and Gawith (1999) (Terminal spikelet } \rightarrow \text { Anthesis } \\
\text { Assumption } \\
\text { Porter and Gawith (1999) (Terminal spikelet } \rightarrow \text { Anthesis } \\
\text { Assumption } \\
\text { Porter and Gawith (1999) (Anthesis }-1 /+4{ }^{\circ} \mathrm{C} \text { ) } \\
\text { Assumption } \\
\text { Roth (2021) }\end{array}$ \\
\hline \multirow[t]{2}{*}{$\theta_{i}^{\mathrm{PH}}$} & $\mathscr{N}\left(\mu, \sigma^{2}\right)$ & $\begin{array}{l}\text { 2016: } \mu_{t \mathrm{PH}_{\text {start }}}=108 \mathrm{~d}, \sigma_{t \mathrm{PH}_{\text {start }}}=2.8 \mathrm{~d} \\
\text { 2017: } \mu_{t \mathrm{PH}_{\text {start }}}=103 \mathrm{~d}, \sigma_{t \mathrm{PH}_{\text {start }}}=3.0 \mathrm{~d} \\
\text { 2018: } \mu_{t \mathrm{PH}_{\text {start }}}=101 \mathrm{~d}, \sigma_{t \mathrm{PH}_{\text {start }}}=3.1 \mathrm{~d}\end{array}$ & $\begin{array}{l}\text { Kronenberg et al. (2020a) } \\
\text { Kronenberg et al. (2020a) } \\
\text { Roth (2021) }\end{array}$ \\
\hline & & $\begin{array}{l}\text { 2016: } \mu_{t \mathrm{PH}_{\text {stop }}}=165 \mathrm{~d}, \sigma_{t \mathrm{PH}_{\text {stop }}}=2.5 \mathrm{~d} \\
\text { 2017: } \mu_{t \mathrm{PH}_{\text {stop }}}=162 \mathrm{~d}, \sigma_{t \mathrm{PH}_{\text {stop }}}=3.5 \mathrm{~d} \\
\text { 2018: } \mu_{t \mathrm{PH}_{\text {stop }}}=158 \mathrm{~d}, \sigma_{t \mathrm{PH}_{\text {stop }}}=4.0 \mathrm{~d}\end{array}$ & $\begin{array}{l}\text { Kronenberg et al. (2020a) } \\
\text { Kronenberg et al. (2020a) } \\
\text { Roth (2021) }\end{array}$ \\
\hline$u_{i t}$ & $\operatorname{AR}(1)$ & $\rho=0.9, \sigma=0.003 \mathrm{~m} \mathrm{~d}^{-1}$ & Assumption \\
\hline & $\operatorname{AR}(1)$ & $\rho=0.3, \sigma_{m}=0.01 \mathrm{~m}$ & Roth et al. (2020) \\
\hline
\end{tabular}

$\rightarrow$ connects lower and upper limit of cardinal temperature ramps
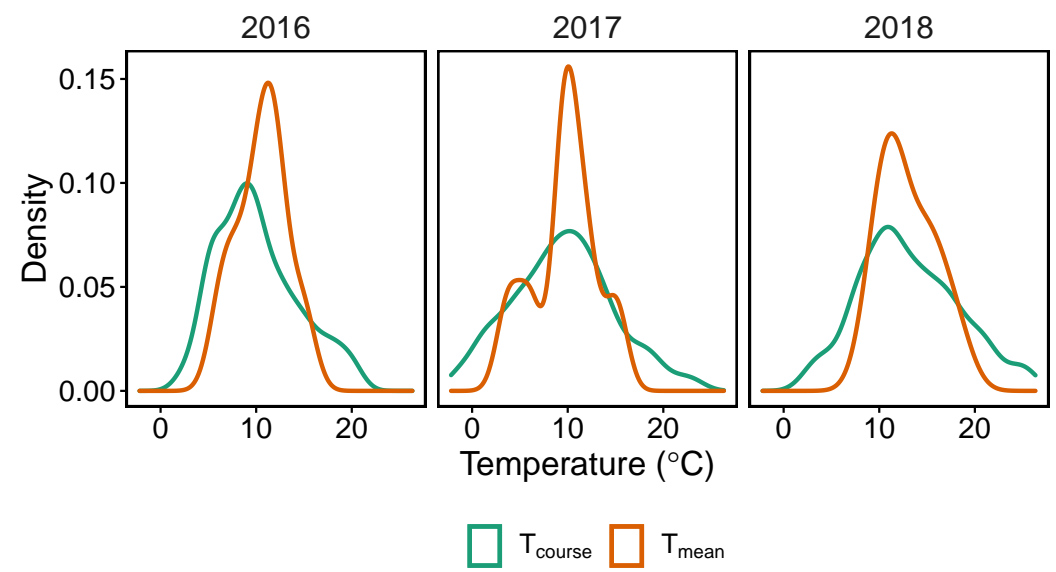

Figure 2: Measured temperatures in the stem elongation phase of winter wheat at the FIP site on an hourly scale $\left(\mathrm{T}_{\text {course }}\right)$ and aggregated to a 3-day scale $\left(\mathrm{T}_{\text {mean }}\right)$. 
As an independent random measurement errors was assumed, the maximum-likelihood optimization parameter $\sigma$ was fixed to the well-known measurement error of the device, herein the simulation input $\sigma_{m}$ (Table 1). Parameters were optimized in R using the method L-BFGS-B of the function optim.

To allow examining the effect of noise caused by inhomogeneities and measurement errors, the extraction was once performed on raw simulated data, once on data contaminated with the measurement error $e$, and once with both measurement and inhomogeneities errors $e$ and $u$. Bias, variance, root-mean squared error (RMSE) and Pearson's correlation were calculated after extracting the parameters $T_{\min }, l r c$ and $r_{\max }$ for the asymptotic model and $a$ (slope) and $T_{\min }$ for the linear model.

\section{Results}

Aggregating measured temperatures at the FIP site to hourly means $\left(T_{\text {course }}\right)$ and 3-day means $\left(T_{\text {mean }}\right)$ reveled sever differences in their distribution (Figure 2): Temperatures close to the cardinal temperatures $T_{\min }$ and $T_{\text {opt }}$ were frequent for $T_{\text {course }}$, but almost absent for $T_{\text {mean }}$. Simulating the stem elongation by combining the $T_{\text {course }}$ measurements with a Wang-Engel dose-response function resulted in an average final height slightly higher than observed for elite varieties under field conditions (Kronenberg et al., 2020b), but realistic individual plant height time series with characteristic starts, stops, and lag phases (Figure 3). Fitting the asymptotic model based on $T_{\text {course }}$ to the simulated data converged for $96 \%$ of all genotypes. All other models converged for all genotypes. In a preliminary run, we also tried to fit the Wang-Engel model to simulated data, but failed to extract meaningful parameters. This was partly due to the complexity of defining valid non-overlapping ranges for the cardinal temperatures, but mainly because the model did not converge in most cases.

Based on the RMSE, the maximum growth rate $r_{\max }$ and base temperature $T_{\min }$ were best estimated with the asymptotic dose-response model (Equation 5 and 6) based on $T_{\text {course }}$ covariates (Table 2). While the variance was drastically lower for both parameters if using $T_{\text {course }}$, the bias slightly increased for $r_{\max }$ but decreased for $T_{\min }$. The RMSE, bias and variance of the linear model for the parameter $T_{\min }$ was much higher than for the asymptotic model.

Introducing a systematic measurement error $e$ did not affect the slope estimated by the linear model; extracted values correlated 1:1 for the simulation with and without noise (Table 3). In contrast, the Pearson's $r$ for $T_{\min }$ was close to zero, but a Spearman's rank correlation revealed nevertheless a high robustness of the extracted ranking to noise. The asymptotic model was more susceptible to noise than the linear model (Table 3). Using temperature courses instead of mean temperatures was of benefit for the parameters $r_{\max }$ and $T_{\min }$, but of disadvantage for lrc.

Correlating simulated versus predicted parameter values revealed further differences between the methods (Figure 4): While the asymptotic model based on $T_{\text {course }}$ was able to extract $r_{\max }$ and $T_{\min }$ with very strong correlations to the input values, the same model based on $T_{\text {mean }}$ yielded only moderate correlations for $r_{\max }$ and $T_{\min }$. 

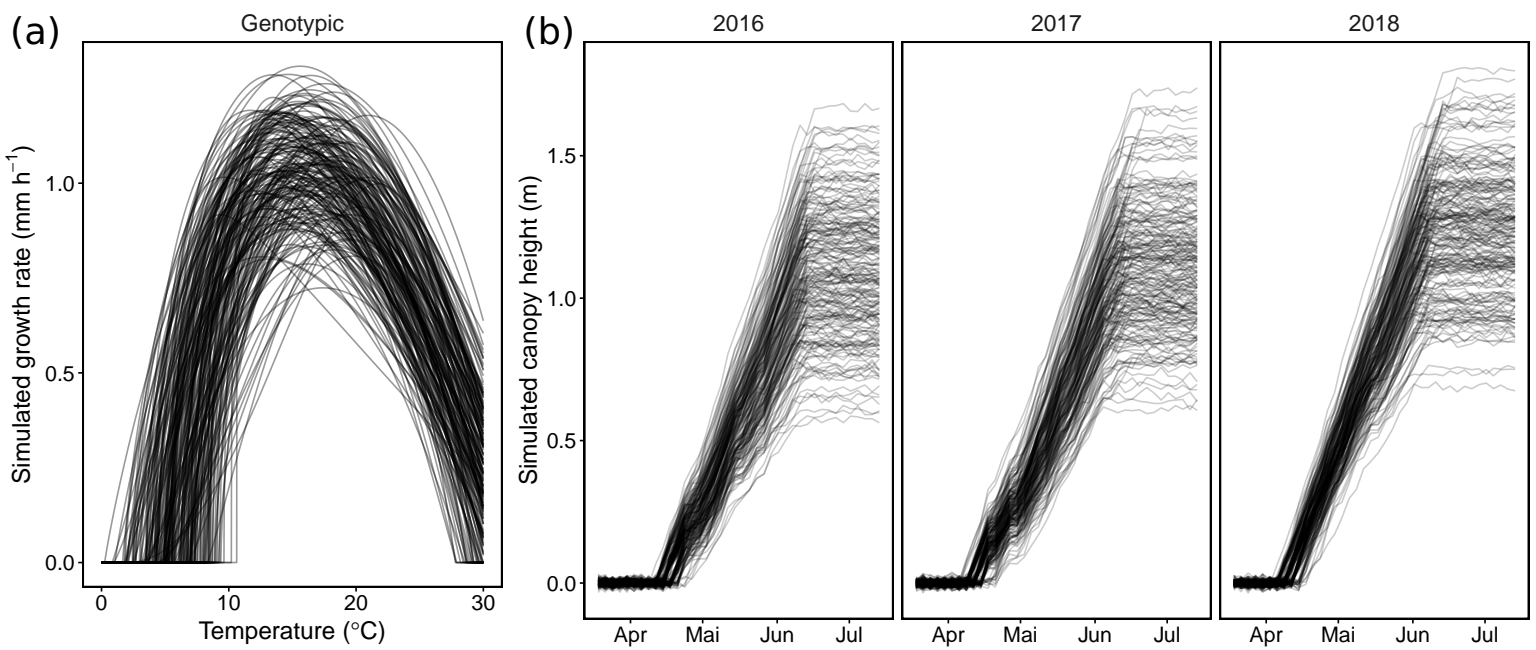

Figure 3: Simulated growth rates (a) and canopy heights (b) for 100 out of 1000 simulated genotypes and three years. Simulated growth rates are based on a Wang-Engel dose-response curve model.

Table 2: Bias, variance and root-mean squared error for the linear and asymptotic model based on mean temperature and temperature courses for simulated data without noise.

\begin{tabular}{lllll}
\hline Parameter & Model & Bias & Var & RMSE \\
\hline$r_{\text {max }}$ & Asymptotic $T_{\text {mean }}$ & 0.0805 & 0.0347 & 0.103 \\
& Asymptotic $T_{\text {course }}$ & 0.0949 & 0.00309 & 0.0959 \\
\hline \multirow{2}{*}{$T_{\text {min }}$} & Linear $T_{\text {mean }}$ & 9.21 & $1.96 e+08$ & 10.6 \\
& Asymptotic $T_{\text {mean }}$ & 0.764 & 2.24 & 1.04 \\
& Asymptotic $T_{\text {course }}$ & -0.352 & 0.575 & 0.439 \\
\hline \multirow{2}{*}{$a$ (slope) } & Linear $T_{\text {mean }}$ & 0.0657 & 0.00272 & 0.0657 \\
\hline
\end{tabular}

Table 3: Pearson's and Spearman's correlation between parameters extracted on simulated data with systematic measurement error noise and without noise.

\begin{tabular}{llll}
\hline Model & Parameter & Pearson's r & Spearman's $r$ \\
\hline Linear $T_{\text {mean }}$ & $a$ (slope) & 1.00 & 1.00 \\
& $T_{\min }$ & 0.00 & 0.88 \\
\hline Asymptotic $T_{\text {mean }}$ & $r_{\max }$ & 0.61 & 0.79 \\
Asymptotic $T_{\text {course }}$ & $r_{\max }$ & 0.85 & 0.92 \\
\hline Asymptotic $T_{\text {mean }}$ & $T_{\min }$ & 0.60 & 0.66 \\
Asymptotic $T_{\text {course }}$ & $T_{\min }$ & 0.77 & 0.72 \\
\hline Asymptotic $T_{\text {mean }}$ & $\operatorname{lrc}$ & 0.69 & 0.73 \\
Asymptotic $T_{\text {course }}$ & $\operatorname{lrc}$ & 0.46 & 0.70 \\
\hline
\end{tabular}


Table 4: Bias, variance, root-mean squared error and Pearson's correlation of the asymptotic model based on temperature courses with simulated noise due to measurement errors and field and environment inhomogeneities.

\begin{tabular}{llllll}
\hline Parameter & Noise & Bias & Var & RMSE & Correlation \\
\hline$r_{\max }$ & - & 0.0949 & 0.00309 & 0.0959 & 0.89 \\
& $e$ & 0.0971 & 0.119 & 0.114 & 0.3 \\
& $e+u$ & 0.0964 & 0.116 & 0.113 & 0.3 \\
\hline \multirow{2}{*}{$T_{\min }$} & - & -0.352 & 0.575 & 0.439 & 0.92 \\
& $e$ & -0.108 & 5.55 & 1.27 & 0.48 \\
& $e+u$ & -0.112 & 5.67 & 1.28 & 0.47 \\
\hline
\end{tabular}

$e:$ systematic measurement error noise

$u$ : field and environment inhomogeneities

The parameter $r_{\max }$ extracted by the asymptotic model based on $T_{\text {mean }}$ was not only correlated with the simulated $r_{\text {max }}$, but also moderately related to $T_{\text {opt }}$. Using $T_{\text {course }}$ reduced this moderate bias for the parameter estimates of $r_{\max }$ but introduced a new weak bias for the parameter estimates of $T_{\min }$ towards $T_{\text {opt }}$. The asymptotic model additionally allowed estimating $T_{\mathrm{opt}}$ using $\operatorname{lrc}$ as proxy with a high correlation to input values.

For the linear model, both parameter estimates of slope and $T_{\min }$ were uncorrelated with the corresponding simulated input parameter (Figure 4b). Instead, the slope was related to $r_{\max }, T_{\min }$ and $T_{\mathrm{opt}}$, while $T_{\min }$ based on the estimated intercept was not correlated to any input parameter. Both the linear and asymptotic model were not affected by supra-optimal temperatures: the input parameter $T_{\max }$ that defined growth in the supra-optimal range above $T_{\mathrm{opt}}$ was uncorrelated with any extracted parameter.

Adding noise to the simulated growth time series led to increased variances for the parameter estimates of $r_{\max }$ and $T_{\min }$ based on the asymptotic model (Table 4). While the RMSE drastically increased by a factor of three for $T_{\min }$ and the correlation to input values dropped below 0.5, it remained low for $r_{\max }$ while the correlation with input parameter dropped to 0.3. The bias for $r_{\max }$ remained unchanged, while the bias for $T_{\min }$ decreased. Adding additional noise caused by highly auto-correlated spatial inhomogeneities did not further decrease the RMSE or increase the variance and bias.

Simulating changing cardinal temperatures for all genotypes alike decreased correlations of extracted parameters for all models (Figure 4c). The differences between $T_{\text {mean }}$ and $T_{\text {course }}$ for the asymptotic model disappeared: While both models were able to extract $r_{\max }$ with moderate correlations, they failed to extract $T_{\min }$. The curvature parameter $\operatorname{lrc}$ was now moderately correlated with $T_{\min }$ and $T_{\text {opt }}$ for $T_{\text {mean }}$ and weakly correlated with $T_{\min }$ and $T_{\text {opt }}$ for $T_{\text {course }}$. The slope extracted by the linear model was moderately related to $T_{\min }$ and weakly to $r_{\max }$ and $T_{\mathrm{opt}}$. Again, $T_{\max }$ was uncorrelated with any extracted parameter.

\section{Discussion}

Although the growth of simulated canopies was based on a Wang-Engel temperature dose-response curve, using a corresponding model to extract parameters turned out to be not suitable, mainly because of failed con- 
bioRxiv preprint doi: https://doi.org/10.1101/2021.07.23.453040; this version posted July 23, 2021. The copyright holder for this preprint (which was not certified by peer review) is the author/funder. All rights reserved. No reuse allowed without permission.
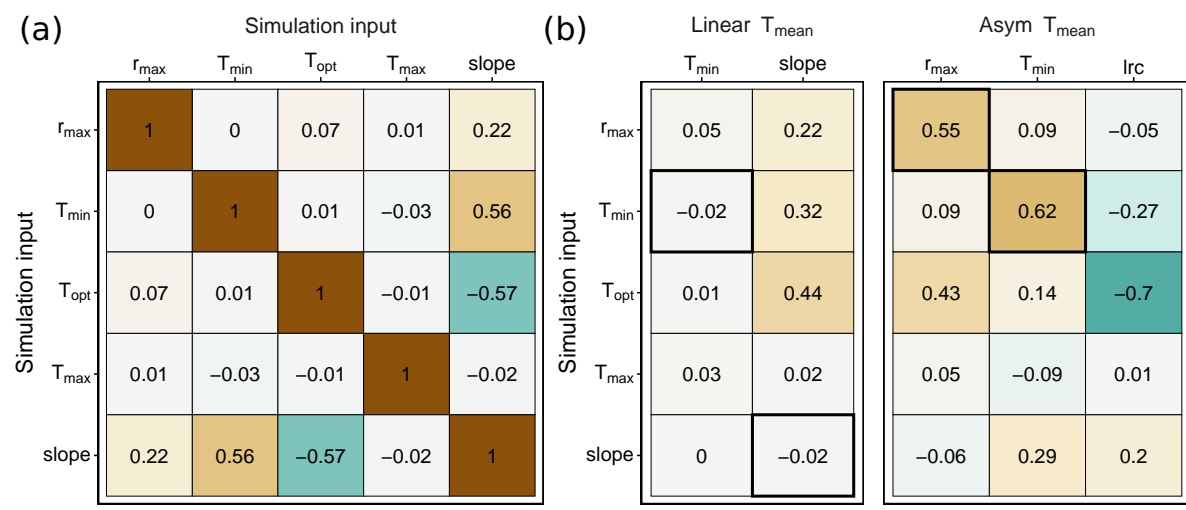

Asym $T_{\text {course }}$
\begin{tabular}{|c|c|c|}
\hline \multicolumn{1}{|c|}{$r_{\text {max }}$} & $T_{\text {min }}$ & Irc \\
\hline 0.89 & 0.01 & -0.05 \\
\hline-0.14 & 0.92 & 0.29 \\
\hline 0.29 & 0.28 & -0.64 \\
\hline 0.17 & -0.13 & -0.08 \\
\hline-0.04 & 0.37 & 0.58 \\
\hline
\end{tabular}

(c)

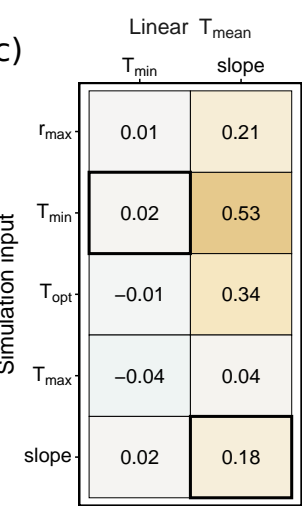

Asym $T_{\text {mean }}$
\begin{tabular}{|c|c|c|}
\hline$r_{\text {max }}$ & \multicolumn{1}{|c|}{$T_{\text {min }}$} & Irc \\
\hline 0.51 & -0.02 & -0.06 \\
\hline 0.19 & 0.03 & -0.45 \\
\hline 0.03 & -0.11 & -0.4 \\
\hline 0.13 & -0.01 & -0.02 \\
\hline 0.21 & 0.07 & -0.07 \\
\hline
\end{tabular}

Asym $T_{\text {course }}$
\begin{tabular}{|c|c|c|}
\hline$r_{\max }$ & $T_{\min }$ & Irc \\
\hline 0.49 & 0 & 0 \\
\hline 0.12 & 0.16 & -0.37 \\
\hline 0.07 & -0.24 & -0.26 \\
\hline 0.11 & -0.01 & -0.02 \\
\hline 0.12 & 0.27 & -0.08 \\
\hline
\end{tabular}

Figure 4: Pearson's correlations of simulated data versus extracted temperature response parameters. Provided are results for the simulation without noise (a) versus extracted temperature response parameters with fixed cardinal temperatures (b) and changing cardinal temperatures with time (c) for the linear model based on mean temperature (Linear $T_{\text {mean }}$ ) and the asymptotic growth model based on mean temperature (Asym $T_{\text {mean }}$ ) and temperature courses (Asym $T_{\text {course }}$ ). Note that the slope is derived from the relation slope $=r_{\text {max }} / T_{\text {opt }}-T_{\text {min }}$ and therefore not an independent input parameter of the simulation. Black bold boxes indicate correlations between predicted and true values for identical parameters. 
vergence. Presumably, non-existing measurements at supra optimal temperatures ( $\gtrsim 30^{\circ} \mathrm{C}$, Figure 2 ) prevented estimating the required parameter $T_{\max }$. As suspected, the asymptotic model performed better with percentages of converged time series close to $100 \%$. Using temperature courses instead of aggregated mean values allowed estimating independent parameters with higher correlations to input parameters, to the cost of a slightly decreased convergence rate. Especially the parameter $T_{\min }$ profited from taking temperature courses into account (Table 2 ), which indicates that in the early phase where stem elongation starts the distribution of temperatures and therefore temperature courses are essential to describe temperature responses ( $\lesssim 5^{\circ} \mathrm{C}$, Figure 2$)$. Additionally to $T_{\min }$, the asymptotic model will extract the maximum growth rate $r_{\max }$ and the parameter $\operatorname{lrc}$ which is related to the optimum temperature $T_{\mathrm{opt}}$, and therefore allow to fully describe temperature responses between the lower base temperature $T_{\min }$ and optimum temperature $T_{\mathrm{opt}}$. A limitation of the proposed asymptotic approach may be the high optimum temperatures: Although the extracted base temperature $T_{\min }$ and maximum growth rate $r_{\max }$ were strongly correlated with input parameters, they were also slightly biased by the optimum temperature $T_{\text {opt }}$.

Noise and environment inhomogeneities reduced the correlation to input parameters drastically, independent on whether RMSE stayed stable or not (Table 4). It seems reasonable to assume that this reduction in correlation will lead to reduced heritabilities of measured traits in HTFP. The simulation was not including a randomized field experiment set-up, but only independent genotype time series. Nevertheless, we assume based on our previous study on two other intermediate traits (Roth et al., 2021) that also for dose-response curve traits it is essential to follow the three principles of experimental design, i.e., replication, randomization and blocking (Kempton, 1984). Such an experimental design will allow to process the extracted plot-based parameters using, e.g., linear mixed models to compensate for measurement errors and environment inhomogeneities and therefore ensure the overall heritability.

Simulating changing cardinal temperatures drastically reduced the ability of the asymptotic model to extract meaningful cardinal temperatures (Figure 4c). We suspect that this effect was overamplified by the simulation and that supposed cardinal temperature ramps in Porter and Gawith (1999) may be too extreme for wheat elite cultivar sets. Nevertheless, in the worst case that cardinal temperature ramp assumptions were justified, the asymptotic model would yield less heritable parameters than in the optimum case of fixed cardinal temperatures, but not introduce additional biases.

In contrast, one may conclude that using a 'simple' linear model to avoid the complexity of curvy doseresponse models is not advisable. The results of this study demonstrated that the extracted linear response to temperature (slope) was similarly related to both cardinal temperatures $T_{\min }$ and $T_{\text {opt }}$ but also to the maximum growth rate $r_{\max }$, while unrelated to the simulated slope (Figure $4 \mathrm{~b}$ ). Thus, the apparent temperature response is mainly driven by cardinal temperatures. When fitting a linear model to a wide temperature range, with significant number of hours in temperature ranges beyond the optimum (Figure 2), it will inevitably compensate for both the maximum growth rate at the optimum and the base temperature of growth. Using a linear model if cardinal temperatures are not fixed is even more harmful: The relation of the slope may switch from $T_{\text {opt }}$ towards 
$T_{\min }$ (Figure 4c). Depending on the examined genetic material, applying a linear model may therefore lead to completely different conclusions. Eventually, one has to review existing literature that refers to slopes as temperature responses (e.g., Kronenberg et al., 2020a) being at least partly affected by growth at optimum temperature, maximum growth rate, and changing cardinal temperatures.

Using modeling we aimed to simulate different aspects of temperature-response during the important phase of stem elongation of wheat. A major advantage of this approach is that it can be used to test different approaches to parameterize temperature-response. This approach is particularly valuable as other factors such as, e.g., the effect of drying soil, extremely low vapor pressure deficit, or low radiation, do not have to be included. For real field data, such influences will have to be considered to improve the model.

\section{Conclusion}

Adequate models to quantify the different aspects of growth response to temperature may greatly improve our understanding of crop adaptation to certain climatic scenarios. Asymptotic models represent a valid choice if sampling winter wheat genotypes in an environment where the decrease in growth after reaching an optimum temperature is negligible. Using temperature courses with high temporal resolution as covariate input for the model fit instead of aggregated mean temperatures is advisable. Changing cardinal temperatures-related to advancing phenology — may reduce the ability to extract parameters with an asymptotic model but will not severely bias results. Nevertheless, measurement noise and environment and field inhomogeneities may significantly increase the variance of extracted parameters. Care should be taken if interpreting existing literature based on linear temperature growth response models, as extracted response reactions may be strongly biased by base and optimum temperature, maximum growth rate at optimum temperature, and changing cardinal temperatures with time. As supra optimal temperatures before flowering are hardly ever reached in a temperate climate, we conclude that models including maximal temperatures will neither deliver any benefit under these conditions.

\section{Acknowledgement}

We like to acknowledge Lukas Kronenberg for feedback on an early version of the manuscript, and Norbert Kirchgessner for feedback on formula notations (both ETH Zurich).

\section{Funding}

LR received funding from Innosuisse (http://www . innosuisse. ch) in the framework for the project "Trait spotting" (grant number: KTI P-Nr 27059.2 PFLS-LS). HPP was supported by DFG grant PI 377/24-1.

\section{Declaration of Competing Interest}

The authors declare no conflict of interest. 
Lukas Roth: Conceptualization, Methodology, Software, Formal analysis, Visualization, Writing - Original Draft. Hans-Peter Piepho: Conceptualization, Methodology, Writing - Review \& Editing. Andreas Hund: Conceptualization, Supervision, Project administration, Funding acquisition, Writing - Review \& Editing.

\section{Data availability}

Data and source code that support the findings of this study are openly available in the ETH gitlab repository at https://gitlab.ethz.ch/crop_phenotyping/htfp_data_processing and archived in the ETH research collection (http://doi.org/10.5905/ethz-1007-385). 
bioRxiv preprint doi: https://doi.org/10.1101/2021.07.23.453040; this version posted July 23, 2021. The copyright holder for this preprint (which was not certified by peer review) is the author/funder. All rights reserved. No reuse allowed without permission.

\section{References}

José Luis Araus, Shawn C. Kefauver, Mainassara Zaman-Allah, Mike S. Olsen, and Jill E. Cairns. Translating High-Throughput Phenotyping into Genetic Gain. Trends in Plant Science, 23(5):451-466, 2018. ISSN 13601385. doi: 10.1016/j.tplants.2018.02.001.

Raymond Bonhomme. Bases and limits to using 'degree.day' units. European Journal of Agronomy, 13:1-10, 2000. ISSN 11610301. doi: 10.1016/S1161-0301(00)00058-7.

Nadine Brisson, Philippe Gate, David Gouache, Gilles Charmet, François Xavier Oury, and Frédéric Huard. Why are wheat yields stagnating in Europe? A comprehensive data analysis for France. Field Crops Research, 119:201-212, 2010. ISSN 03784290. doi: 10.1016/j.fcr. 2010.07.012.

José A. Clavijo Michelangeli, Thomas R. Sinclair, and Nikolay Bliznyuk. Using an Arrhenius-type function to describe temperature response of plant developmental processes: Inference and cautions. New Phytologist, 210(2):377-379, 2016. ISSN 14698137. doi: 10.1111/nph. 13812.

Christoph Grieder, Andreas Hund, and Achim Walter. Image based phenotyping during winter: a powerful tool to assess wheat genetic variation in growth response to temperature. Functional Plant Biology, 42:387-396, 2015. ISSN 1445-4408. doi: 10.1071/fp14226.

Andreas Hund, Regina Reimer, Peter Stamp, and Achim Walter. Can we improve heterosis for root growth of maize by selecting parental inbred lines with different temperature behaviour? Philosophical Transactions of the Royal Society B: Biological Sciences, 367:1580-1588, 2012. ISSN 14712970. doi: 10.1098/rstb.2011.0242.

Frank H. Johnson, Hentry Eyring, and R. W. Williams. The nature of enzyme inhibitions in bacterial luminescence: sulfanilamide, urethane, temperature and pressure. Journal of Cellular and Comparative Physiology, 20(3):247-268, 1942.

R.A. Kempton. The design and analysis of unreplicated field trials. Vorträge für Pflanzenzüchtung, 7:219-242, 1984.

Lukas Kronenberg, Kang Yu, Achim Walter, and Andreas Hund. Monitoring the dynamics of wheat stem elongation: genotypes differ at critical stages. Euphytica, 213(157), 2017. doi: 10.1007/s10681-017-1940-2.

Lukas Kronenberg, Steven Yates, Martin P Boer, Norbert Kirchgessner, Achim Walter, and Andreas Hund. Temperature response of wheat affects final height and the timing of stem elongation under field conditions. Journal of Experimental Botany, 2020a. doi: 10.1093/jxb/ eraa471.

Lukas Kronenberg, Steven Yates, Shiva Ghiasi, Lukas Roth, Michael Friedli, Michael E. Ruckle, Roland A. Werner, Flavian Tschurr, Melanie Binggeli, Nina Buchmann, Bruno Studer, and Achim Walter. Rethinking temperature effects on leaf growth, gene expression and metabolism: Diel variation matters. Plant, Cell and Environment, pages 1-15, 2020b. ISSN 0140-7791. doi: 10.1111/pce.13958.

Friedrich Laidig, Hans-Peter Piepho, Dirk Rentel, Thomas Drobek, Uwe Meyer, and Alexandra Huesken. Breeding progress, environmental variation and correlation of winter wheat yield and quality traits in German official variety trials and on-farm during 1983-2014. Theoretical and Applied Genetics, 130:223-245, 2017. doi: 10.1007/s00122-016-2810-3.

C. E. Timothy Paine, Toby R. Marthews, Deborah R. Vogt, Drew Purves, Mark Rees, Andy Hector, and Lindsay A. Turnbull. How to fit nonlinear plant growth models and calculate growth rates: An update for ecologists. Methods in Ecology and Evolution, 3:245-256, 2012. ISSN 2041210X. doi: 10.1111/j.2041-210X.2011.00155.x.

Boris Parent and François Tardieu. Temperature responses of developmental processes have not been affected by breeding in different ecological areas for 17 crop species. New Phytologist, 194:760-774, 2012. ISSN 0028646X. doi: 10.1111/j.1469-8137.2012.04086.x.

Boris Parent, O. Turc, Y. Gibon, M. Stitt, and F. Tardieu. Modelling temperature-compensated physiological rates, based on the co-ordination of responses to temperature of developmental processes. Journal of Experimental Botany, 61(8):2057-2069, 2010. ISSN 00220957. doi: $10.1093 /$ jxb/erq003.

Boris Parent, Emilie J. Millet, and François Tardieu. The use of thermal time in plant studies has a sound theoretical basis provided that confounding effects are avoided. Journal of Experimental Botany, 70(9):2359-2370, 2019. ISSN 14602431. doi: 10.1093/jxb/ery402.

John R. Porter and Megan Gawith. Temperatures and the growth and development of wheat a review. European Journal of Agronomy, 10: 23-36, 1999. doi: 10.1016/S1161-0301(98)00047-1.

Julian Ramirez-Villegas, James Watson, and Andrew J. Challinor. Identifying traits for genotypic adaptation using crop models. Journal of Experimental Botany, 66(12):3451-3462, 2015. ISSN 14602431. doi: 10.1093/jxb/erv014.

François Rebaudo and Victor Badre Rabhi. Modeling temperature-dependent development rate and phenology in insects: review of major developments, challenges, and future directions. Entomologia Experimentalis et Applicata, 166:607-617, 2018. ISSN 15707458. doi: 10.1111 /eea.12693.

François Rebaudo, Quentin Struelens, and Olivier Dangles. Modelling temperature-dependent development rate and phenology in arthropods: The devRate package for R. Methods in Ecology and Evolution, 9:1144-1150, 2018. ISSN 2041210X. doi: 10.1111/2041-210X.12935.

G. J. Rebetzke, J. Jimenez-Berni, R. A. Fischer, D. M. Deery, and D. J. Smith. Review: High-throughput phenotyping to enhance the use of crop genetic resources. Plant Science, 282:40-48, 2019. doi: 10.1016/j.plantsci.2018.06.017.

Regina Reimer, Benjamin Stich, Albrecht E. Melchinger, Tobias A. Schrag, Anker P. Sørensen, Peter Stamp, and Andreas Hund. Root response to temperature extremes: Association mapping of temperate maize (Zea mays L). Maydica, 58:156-168, 2013. ISSN 22798013.

Lukas Roth. Development of drone-based phenotyping methodologies to support physiological plant breeding of wheat and soybean. PhD thesis, mar 2021. URL https: //www.research-collection.ethz.ch:443/handle/20.500.11850/474271.

Lukas Roth, Moritz Camenzind, Helge Aasen, Lukas Kronenberg, Christoph Barendregt, Karl-Heinz Camp, Achim Walter, Norbert Kirchgessner, and Andreas Hund. Repeated Multiview Imaging for Estimating Seedling Tiller Counts of Wheat Genotypes Using Drones. Plant Phenomics, 2020(3729715), 2020. doi: 10.34133/2020/3729715.

Lukas Roth, María Xosé Rodríguez-Álvarez, Fred van Eeuwijk, Hans-Peter Piepho, and Andreas Hund. Phenomics data processing: A plot-level model for repeated measurements to extract the timing of key stages and quantities at defined time points. bioRxiv, page 2021.05.02.442243, jan 2021. doi: 10.1101/2021.05.02.442243.

Gustavo A. Slafer and H. M. Rawson. Rates and cardinal temperatures for processes of development in wheat: Effects of temperature and thermal amplitude. Australian Journal of Plant Physiology, 22:913-926, 1995. ISSN 03107841. doi: 10.1071/PP9950913.

Fred A. van Eeuwijk, Daniela Bustos-Korts, Emilie J. Millet, Martin P. Boer, Willem Kruijer, Addie Thompson, Marcos Malosetti, Hiroyoshi 
bioRxiv preprint doi: https://doi.org/10.1101/2021.07.23.453040; this version posted July 23, 2021. The copyright holder for this preprint (which was not certified by peer review) is the author/funder. All rights reserved. No reuse allowed without permission.

Iwata, Roberto Quiroz, Christian Kuppe, Onno Muller, Konstantinos N. Blazakis, Kang Yu, Francois Tardieu, and Scott C. Chapman. Modelling strategies for assessing and increasing the effectiveness of new phenotyping techniques in plant breeding. Plant Science, 282: 23-39, 2019. ISSN 18732259. doi: 10.1016/j.plantsci.2018.06.018.

R. van Haren. Evaluation of crop production sensitivity to weather extremes. Workplan Document of the Research Institute for Agrobiology and Soil Fertility and the C.T. de Wit Graduate School for Production Ecology. Technical report, 1996.

Enli Wang and Thomas Engel. Simulation of phenological development of wheat crops. Agricultural Systems, 58(1):1-24, 1998. ISSN 0308521X. doi: 10.1016/S0308-521X(98)00028-6.

Enli Wang, Pierre Martre, Zhigan Zhao, Frank Ewert, Andrea Maiorano, Reimund P. Rötter, Bruce A. Kimball, Michael J. Ottman, Gerard W. Wall, Jeffrey W. White, Matthew P. Reynolds, Phillip D. Alderman, Pramod K. Aggarwal, Jakarat Anothai, Bruno Basso, Christian Biernath, Davide Cammarano, Andrew J. Challinor, Giacomo De Sanctis, Jordi Doltra, Elias Fereres, Margarita Garcia-Vila, Sebastian Gayler, Gerrit Hoogenboom, Leslie A. Hunt, Roberto C. Izaurralde, Mohamed Jabloun, Curtis D. Jones, Kurt C. Kersebaum, Ann Kristin Koehler, Leilei Liu, Christoph Müller, Soora Naresh Kumar, Claas Nendel, Garry O’Leary, Jørgen E. Olesen, Taru Palosuo, Eckart Priesack, Ehsan Eyshi Rezaei, Dominique Ripoche, Alex C. Ruane, Mikhail A. Semenov, Iurii Shcherbak, Claudio Stöckle, Pierre Stratonovitch, Thilo Streck, Iwan Supit, Fulu Tao, Peter Thorburn, Katharina Waha, Daniel Wallach, Zhimin Wang, Joost Wolf, Yan Zhu, and Senthold Asseng. The uncertainty of crop yield projections is reduced by improved temperature response functions. Nature Plants, 3(17102), 2017. ISSN 20550278. doi: 10.1038/nplants.2017.102. 